\title{
Association Between the Rates of Multiple Maternities
}

\author{
Johan Fellman and Aldur W. Eriksson \\ Folkhälsan Institute of Genetics, Population Genetics Unit, Finland
}

W analyze the association between the rates of multiple maternities. Correlation analysis is suitable if there are at least monotonic relationships between the variables. A decreasing tendency can be observed in the rates of multiple maternities in Sweden and in its 25 counties for the period 1751-1960 and the effect of external variables can be assumed to be monotonic. After 1960, the rates of multiple maternities show marked increases, mainly caused by ovulation stimulants and the introduction of other artificial reproduction techniques. We transformed the triplet and quadruplet rates according to Hellin's law in order to obtain rates on the same scale as the twinning rate. The time trends for the multiple maternities in Sweden as a whole were quite similar. The regional differences in the twinning rates could not be satisfactorily explained by maternal age. Alternative attempts to eliminate the effect of other time-dependent factors were to study partial correlation coefficients when the time was kept fixed and the correlation coefficients based on cross-sectional regional data. Both the ordinary and the partial correlation coefficients showed strong regional variation. Cross-sectional analyses gave correlation coefficients similar to the partial correlation coefficients for Sweden as a whole. The variations in the correlation coefficients between the twinning and the triplet rates seem to be caused by other time-dependent factors and the effects of these factors show strong regional variation. After elimination of such factors, the correlation between the twinning and the triplet rates is moderate.

In earlier studies it has been noted that for populations and periods with high twinning rates $(W)$ the rates of higher multiple maternities are also high (Eriksson, 1973; Eriksson \& Fellman, 1973). In this study we analyze the association between the rates of multiple maternities based on both ordinary and partial correlation coefficients, which are suitable if there are approximately linear or at least monotonic relationships between the variables. We present transformations of the correlation coefficients in order to introduce tests and confidence intervals (CI) for them.
In order to eliminate the effect of time-dependent factors, we also study the partial correlation coefficients between the twinning and the triplet rates when time is kept fixed and the correlation coefficients for cross-sectional data. We analyze the association between the rates of multiple maternities in Sweden and in its 25 counties. In order to pursue the monotonicity in these secular trends, we consider only the period 1751-1960. After the 1780s, particularly, a general decreasing tendency can be observed in Sweden and, consequently, the effect of external variables can be assumed to be monotonic. After 1960, the rates of the multiple maternities show marked increases caused by the increased use of fertility drugs and in-vitro fertilization. These increases are more accentuated for the triplet $(R)$ and quadruplet $(Q)$ rates than for the $W$. In order to obtain the same scale and, consequently, to improve the comparisons between the $W$ and the $R$, we present some general statistical results concerning variable transformations and apply transformations based on Hellin's law (Eriksson \& Fellman, 2004; Fellman \& Eriksson, 1993; Hellin, 1895). In recent papers (Eriksson \& Fellman, 2004; Fellman \& Eriksson, 2003), we have compared the temporal trends in the $W$ and the $R$ for Sweden since 1751. The trends in the $W, R$ and $Q$ rates are presented in Figure 1. The general pattern of the curves is rather similar. However, the minimum quadruplet rate appears in the period 1931-1960. In fact, during the whole period 1931-1955 no quadruplets were registered. We also stressed that the strong secular and regional variations in the rates could not be satisfactorily explained by maternal age. The ordinary correlation coefficients between time, the $W$ and the $R$ showed strong regional variation. The same was observed when we eliminated the time and considered the partial correlation coefficients between the $W$ and the $R$. In an alternative attempt to eliminate

Received 16 February, 2004; accepted 10 June, 2004

Address for correspondence: Professor Johan Fellman, Folkhälsan Institute of Genetics, Population Genetics Unit, POB 211, FIN-00251 Helsinki, Finland.E-mail: johan.fellman@shh.fi 


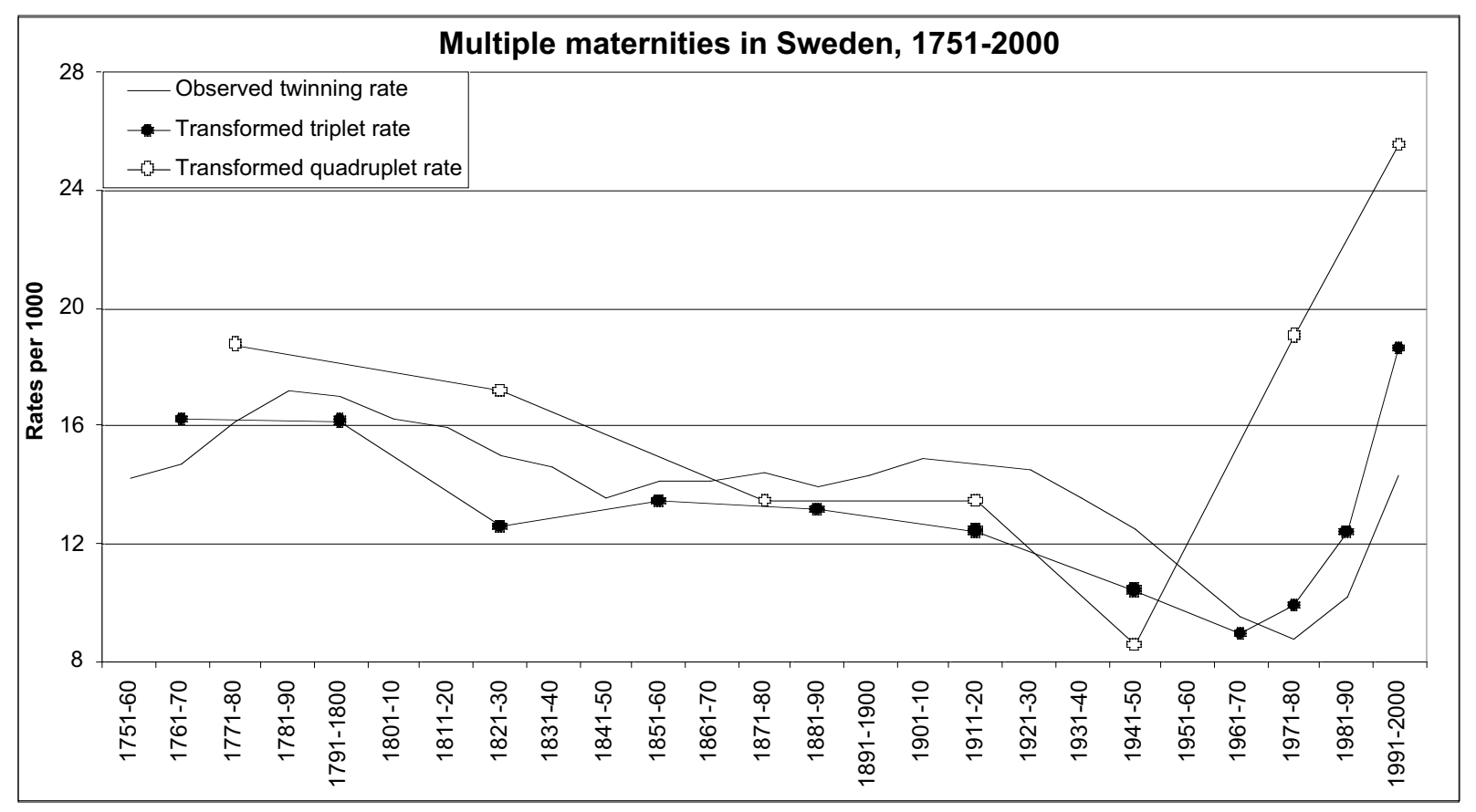

\section{Figure 1}

Temporal variation in the twinning rate and in the transformed triplet and quadruplet rates for Sweden, 1751-2000.

The twinning rates are given for 10-year periods and the triplet rates for 30-year periods up to 1960 and after that for 10-year periods, and the quadruplet rates for 50-year periods up to 1900 and after that for three 30-year periods and one 10-year period.

the time dependency, we also considered the correlations obtained in the cross-sectional regional data. Therefore our opinion is that the variations are caused by other influential, time-dependent factors and that the effects of these factors show strong regional variation. Our general conclusion is that, after elimination of exogenous factors, the correlation between $W$ and $R$ is moderate.

\section{Material}

We analyzed demographic data, pooled for 10-year periods, between 1751 and 2000 in Sweden as a whole (in short, Sweden) and to 1960 in its 25 counties. The Swedish data consist of $25,567,357$ maternities, including 354,083 twin pairs, 4721 triplet sets and 112 quadruplet sets. For some counties the annual twinning data are occasionally missing and therefore, in the study of the regional variation the whole year for such counties is ignored. In spite of this, we consider that the available data represent whole decades. A detailed description of the available data is given in Fellman and Eriksson (2003).

\section{Methods and Results \\ Hellin's Formula and Triplet Maternities}

In Fellman and Eriksson (1993) we stressed that, if one has to compare temporal or regional variation in the rates of twin, triplet and quadruplet maternities, reliable results are obtained if the rates are transformed according to Hellin's law (Hellin, 1895).
Although this law is by no means mathematically exact, the transformed rates are all on the same scale (Fellman \& Eriksson, 1993). Hellin's law can be considered as a simple probability model for the ovulation of additional eggs. However, the discrepancies in the observed twinning rates and the transformed rates of triplets can, to some extent, be caused by temporal and regional changes in the population structure, with breaking up of the old static agrarian society as a consequence of increasing industrialization and urbanization. It seems that the triplet and especially the quadruplet rates in Sweden have in earlier times been considerably higher than Hellin's empirical law predicts. In Hellin's simple formula no account is taken of the different zygosity types in the sets of multiple maternities and the variation in maternal age and parity. Nevertheless, the formula appears to provide a relatively good rule of thumb for the order of magnitude of rates of multiple maternities in Sweden at least up to the 20th century (Figure 1).

In the literature, one can find several attempts to improve Hellin's law. Allen (1960) has proposed one method based on the zygosity of the twin pairs and the triplet sets. Bulmer (1970) has later discussed this method. This method requires information about the sexes of the twin pairs and the triplet sets. In our study of multiple maternities in Sweden, we have information about the sex composition of the twin pairs and the triplet sets for the period 1869-1878 (Berg, 1880) and decade data for the period 
1901-1960. Following the model proposed, we apply Weinberg's law and obtain the dizygotic $(D)$ and monozygotic $(M)$ twinning rates. We also introduce the numbers of unlike-sexed $(U)$ and like-sexed $(L)$ triplet sets. According to the Allen-Bulmer model, the rate of dizygotic triplets is $2 M D$ and the total number of dizygotic $\left(T_{2}\right)$ triplets is $2 N M D$ where $N$ is the total number of maternities. The total number of trizygotic triplet sets is

$T_{3}=\frac{4}{3}\left(U-1 / 2 T_{2}\right)$

and the number of monozygotic triplet sets is $T_{1}=L-\frac{1}{3} U-\frac{1}{3} T_{2}$

Finally, it was assumed that the total number of monozygotic triplet sets is proportional to $M^{2}$ and the number of trizygotic triplet sets is proportional to $D^{2}$. Consequently, the improved Hellin's formula is $R=k_{1} M^{2}+2 M D+k_{3} D^{2}$. For $k_{1}=k_{3}=1$, the Hellin's law is obtained. Based on empirical data the factors $k_{1}$ and $k_{3}$ should be estimated by the formulae

$k_{1}=\frac{\text { total number of monozygotic twins }}{\sum N_{i} M_{i}^{2}}$

and

$k_{3}=\frac{\text { total number of dizygotic twins }}{\sum N_{i} D_{i}^{2}}$

Using data from England and Wales, USA, and Italy, Bulmer (1970) obtained the empirical formula $R=1.36 M^{2}+2 M D+0.47 D^{2}$. This formula can be written $R=1.36 M^{2}+2 M D+0.47 D^{2}=M^{2}+2 M D+$ $D^{2}+0.36 M^{2}-0.53 D^{2}=(M+D)^{2}+0.36 M^{2}-0.53 D^{2}$ $=W^{2}+0.36 M^{2}-0.53 D^{2}$.

With respect to Hellin's law, the correction is $0.36 \mathrm{M}^{2}-0.53 \mathrm{D}^{2}$. This is negative if $\mathrm{D} \geq 0.82 \mathrm{M}$. The dizygotic rate $D$ is almost always greater than the monozygotic rate $M$ and, consequently, a deficit in the triplet rate in comparison with Hellin's law is common. In our study of the multiple maternities in Sweden, we have information about the sex composition of the twin pairs and the triplet sets for the periods introduced above. Numerically, we obtain the estimated model $R=1.39 M^{2}+2 M D+0.44 D^{2}$. The parameter estimates differ only slightly from Bulmer's and we observe also that in our result $k_{1}>1$ and $k_{3}<1$. According to our formula, a deficit of triplet sets compared with Hellin's law is obtained if $\mathrm{D} \geq$ $0.84 \mathrm{M}$. In fact, according to the data analyzed, $\mathrm{D} \approx$ $2.89 \mathrm{M}$ and the deficit is obvious. Our results are presented graphically in Figure 2. In this figure we include the estimated $R$ according to both Bulmer's and our parameter estimates.

\section{Transformation of Random Variables}

General. Our study of transformed variables is based on the following general analysis. Consider a stochastic variable $X$ with the mean $\mu_{X}$ and the variance $\sigma_{X}^{2}$. For the transformed variable $Y=u(X)$, approximate formulae for $\mu_{\mathrm{Y}}$ and $\sigma_{Y}^{2}$ can be obtained in the follow-

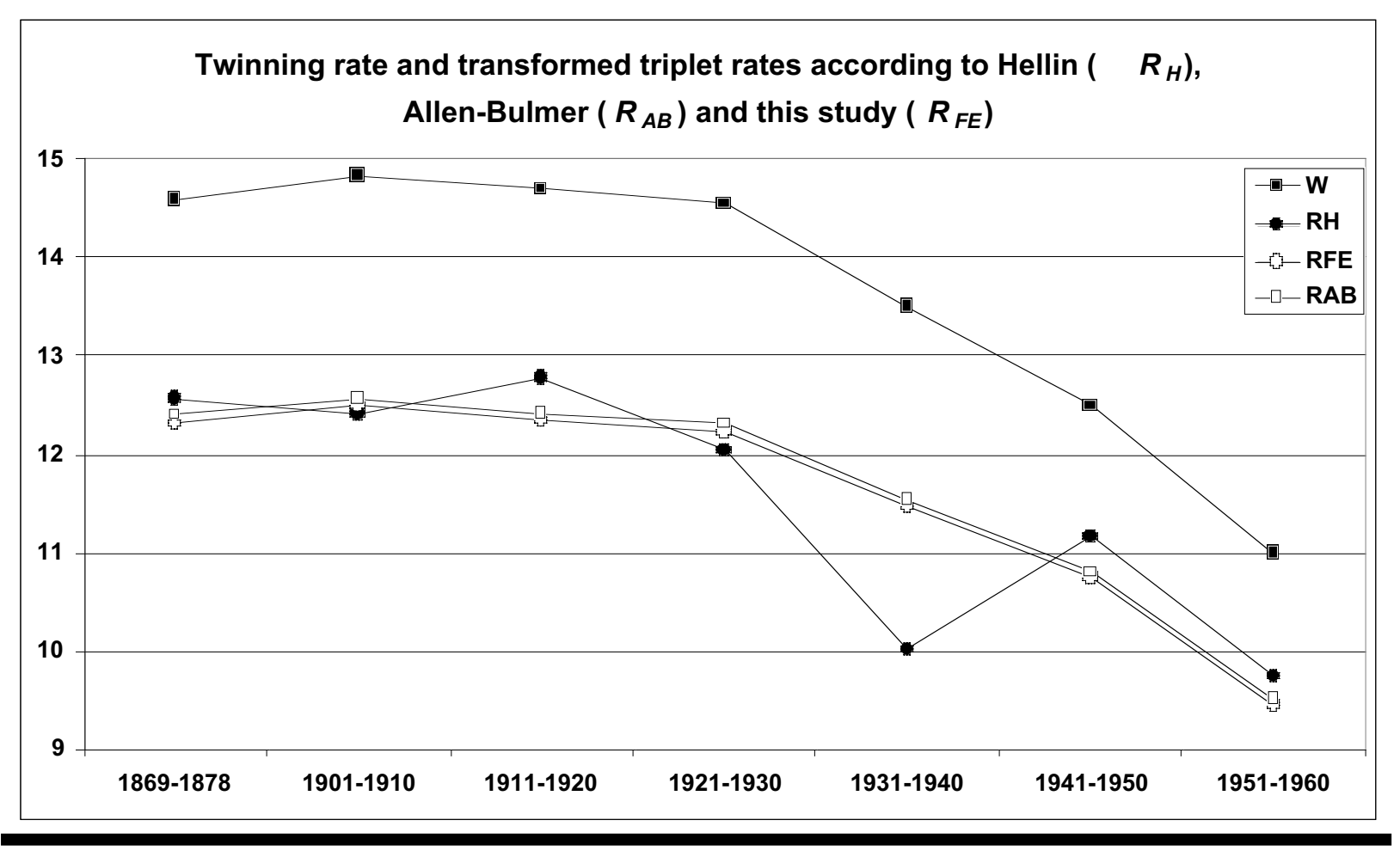

\section{Figure 2}

Graphical presentation of the twinning rate $(W)$, the triplet rate transformed according to Hellin's law $\left(R_{H}\right)$, according to the estimated AllenBulmer model $\left(R_{A B}\right)$ and according to our estimated model $\left(R_{F E}\right)$.

In order to obtain all the curves on the same scale, the square roots of the estimated values are presented. 
ing way. If one takes the first two terms of the Taylor series of $Y=u(X)$ at the mean $\mu_{X}$, one gets

$Y=u(X) \approx u\left(\mu_{X}\right)+\left(\frac{d u}{d x}\right)_{x=\mu_{X}}\left(X-\mu_{X}\right)$

Consequently,

$E(Y)=E(u(X)) \approx u\left(\mu_{X}\right)$ and $\operatorname{Var}(Y) \approx\left(\left(\frac{d u}{d x}\right)_{x=\mu_{X}}\right)^{2} \sigma_{X}^{2}$

Assume that an unknown parameter is $\theta$ and the estimator is $\hat{\theta}$. Using the variable transformations presented above, a transformed parameter $\omega=u(\theta)$ has the estimator $\hat{\omega}=u(\hat{\theta})$. According to the general theory, it follows that if $\hat{\theta}$ is a maximum likelihood (ML) estimator of $\theta$, then $\hat{\omega}=u(\hat{\theta})$ is also an ML estimator of $\omega=u(\theta)$. The estimator $\hat{\omega}=u(\hat{\theta})$ has the approximate mean $E(\hat{\omega}) \approx u(\theta)$ and variance

$\operatorname{Var}(\hat{\omega}) \approx\left(\left(\frac{d u}{d \theta}\right)\right)^{2} \operatorname{Var}(\hat{\theta})$

Tests of $\theta$ and $\omega=u(\theta)$ and can be based on $\hat{\theta}$ and $\hat{\omega}=u(\hat{\theta})$, respectively. Both $\hat{\theta}$ and $\hat{\omega}$ are asymptotically normal, but if $u(\cdot)$ is nonlinear, both estimators cannot be unbiased.

Transformation of the triplet rate. In this connection we consider the rates in absolute proportions. For the triplet rate one has to apply a square root transformation, $R_{H}=\sqrt{R}$, and for the quadruplet rate the cube root transformation, $Q_{H}=\sqrt[3]{Q}$. In Fellman and Eriksson (1993) we have also proposed the $R_{a}=$ $\arcsin (\sqrt{R})$ transformation instead of the simple square root transformation. The transformed rates are attached with appropriate sub-indices $(H$ for Hellin and $a$ for arcsin). If the general formulae (1) and $(2)$ are applied in this context we obtain

$E\left(\hat{R}_{H}\right) \approx \sqrt{R}$

$\operatorname{Var}\left(\hat{R}_{H}\right) \approx\left(\frac{1}{2 \sqrt{R}}\right)^{2} \frac{R(1-R)}{n}=\frac{1-R}{4 n}$

and

$E\left(\hat{R}_{a}\right) \approx \arcsin (\sqrt{R})$

$\operatorname{Var}\left(\hat{R}_{a}\right) \approx\left(\frac{1}{2 \sqrt{1-R} \sqrt{R}}\right)^{2} \frac{R(1-R)}{n}=\frac{1}{4 n}$

In (3) and (4) $\hat{R}_{H}$ and $\hat{R}_{a}$ are the observed, transformed rates. The triplet rate is small, $R \approx 10^{-4}$ and consequently $\hat{R}_{a}$ and $\hat{R}_{H}$ and the two alternatives give almost similar values. If we compare the variances $\operatorname{Var}\left(\hat{R}_{H}\right)$ and $\operatorname{Var}\left(\hat{R}_{a}\right)$ we observe that the variance formulae are also numerically almost identical, but $\operatorname{Var}\left(\hat{R}_{a}\right)>\operatorname{Var}\left(\hat{R}_{H}\right)$. The arcsin transformation yields a standard deviation (approximately) independent of the initial level of the rate and is therefore proposed in the statistical literature. In this paper, we use the simpler square root transformation.

The estimators $\hat{W}$ and $\hat{R}$ are unbiased ML estimators and approximately normal. The transformed estimators $\hat{R}_{H}$ and $\hat{R}_{a}$ are still ML estimators of $\hat{R}_{H}$ and $\hat{R}_{a}$. They are approximately normal, but biased.

\section{Confidence Intervals}

In general, the confidence interval for an unknown parameter is obtained in the following way. Consider an unknown parameter $\theta$ and its estimate $\hat{\theta}$ with the standard error $\operatorname{SE}(\hat{\theta})$. We assume that $\hat{\theta}$ is asymptotically normal and

$z=\frac{\hat{\theta}-\theta}{S E(\hat{\theta})}$

is asymptotically $N(0,1)$. This is the case when $\hat{\theta}$ is an ML estimator. Based on these results, we obtain for the unknown parameter $\theta$ the approximate $100(1-\alpha)$ per cent confidence interval

$\left(\hat{\theta}-z_{1 / 2 \alpha} S E(\hat{\theta}), \hat{\theta}+z_{1 / 2 \alpha} S E(\hat{\theta})\right)$

where $z_{1 / 2 a}$ is the $(1-1 / 2 \alpha)$ quantile of $z$.

Assume that we have obtained a reliable confidence interval for the initial parameter $\theta$, that is, we can define the limits $\theta_{\mathrm{L}}$ and $\theta_{\mathrm{U}}$, such that

$P\left(\theta_{L}<\theta<\theta_{U}\right)=1-\alpha$

If we assume that the transformation $\omega=u(\theta)$ is monotonic (increasing), then we obtain from (5) the CI formula for $u(\theta)$

$P\left(u\left(\theta_{L}\right)<u(\theta)<u\left(\theta_{U}\right)\right)=1-\alpha$

which is as reliable as (5).

\section{Correlation Analysis}

General results. The testing of the estimated correlation coefficients and the construction of CIs for them are connected with a fundamental problem (for detailed analyses, see Hald, 1967, Ch. 19-20; and Kendall \& Stuart, 1967, Ch. 26). Let the theoretical correlation coefficient be $\rho$ and the estimate $r$. For $\operatorname{Var}(r)$ the simple but approximate formula

$\operatorname{Var}(r)=\frac{1}{n}\left(1-\rho^{2}\right)^{2}$

holds good. If the null hypothesis is $\rho=0$, then the standard test statistic is

$t=\sqrt{n-2} \frac{r}{\sqrt{1-r^{2}}}$

which is approximately $t$ distributed with $n-2$ degrees of freedom. This $t$ test is mathematically equivalent to the $t$ test of the regression coefficient in the simple linear regression model.

However, the estimated correlation coefficient $(r)$ is asymptotically normal only for very large samples $(n$ $>500$ ) and for small values of $\rho$. In order to eliminate this problem, one has to perform the variable transformation described by Kendall and Stuart (1967, Ch. 26). We introduce the transformed variables

$\varsigma=1 / 2 \log \left(\frac{1+\rho}{1-\rho}\right)$

for the theoretical, and

$z=1 / 2 \log \left(\frac{1+r}{1-r}\right)$

for the empirical correlation coefficient. For small $\rho$ and $r, \mathrm{~s} \approx \rho$ and $z \approx r$. The transformed variable $z$ is already asymptotically normal for small samples $(n>$ 
50) and reliable results can be obtained for even smaller samples. If we apply the approximate formula (1), we obtain

$z=1 / 2 \log \left(\frac{1+r}{1-r}\right) \approx 1 / 2 \log \left(\frac{1+\rho}{1-\rho}\right)+\left(\frac{1}{1-\rho^{2}}\right)^{2}(r-\rho)=$

$\varsigma+\left(\frac{1}{1-\rho^{2}}\right)^{2}(r-\rho)$

$E(z) \approx \varsigma$ and $\operatorname{Var}(z) \approx \frac{1}{n}$

However, a more exact analysis gives

$\operatorname{Var}(z) \approx \frac{1}{n-3}$

This variance formula has the property that it is independent of the theoretical but unknown $\rho$. In fact, it can be made even more exact at the expense of the independence of $\rho$ and, when the sample size is small, a more exact formula may be of interest. Although we consider small samples in this study, we note that the variance formula (6) is a slight overestimation and we use it. The formulae obtained and the asymptotic normality of $z$ give accurate tests of the estimated correlation coefficients and rather strict confidence intervals.

The test of a null hypothesis $\rho=\rho_{0} \neq 0$ or alternatively,

$\varsigma=\varsigma_{0}=1 / 2 \log \left(\frac{1+\rho_{0}}{1-\rho_{0}}\right) \neq 0$

is based on the test statistic $Z=\sqrt{n-3} 3\left(z-\varsigma_{0}\right)$, which, under the null hypothesis, is asymptotically $\mathrm{N}(0,1)$.

Following Hald (1967, Ch. 19-20), the comparison of several correlation coefficients can be performed in the following way. Consider a set of $k$ independent sub-samples having the sample sizes $\left(n_{1}\right.$, $\left.n_{2}, \ldots, n_{k}\right)$. Let the theoretical correlation coefficients be $\left(\rho_{1}, \rho_{2}, \ldots, \rho_{k}\right)$ and the estimated ones $\left(r_{1}, r_{2}, \ldots, r_{k}\right)$. Furthermore, let $\left(\varsigma_{1}, \varsigma_{2}, \ldots, \varsigma_{k}\right)$ and $\left(z_{1}, z_{2}, \ldots, z_{k}\right)$ be the corresponding transformed variables. We introduce the weighted mean

$\bar{z}=\frac{\sum\left(n_{i}-3\right) z_{i}}{\sum\left(n_{i}-3\right)}$

Its variance is

$\operatorname{Var}(\bar{z})=\frac{\sum\left(n_{i}-3\right)^{2} \operatorname{Var}\left(z_{i}\right)}{\left(\sum\left(n_{i}-3\right)\right)^{2}} \approx \frac{\sum\left(n_{i}-3\right)}{\left(\sum\left(n_{i}-3\right)\right)^{2}}=\frac{1}{\sum n_{i}-3 k}$

Under the null hypothesis $\rho_{1}=\rho_{2}=\ldots=\rho_{k}=\rho_{0}$, or alternatively $\varsigma_{1}=\varsigma_{2}=\ldots=\varsigma_{k}=\varsigma_{0}$, we obtain $E(\bar{z})=\varsigma_{0}$. The weights in $\bar{z}$ are approximately equal to $\left(\operatorname{Var}\left(z_{i}\right)\right)^{-1}$ and, consequently, the weighted mean is an almost efficient estimate. Under the proposed null hypothesis, the test statistic

$\chi^{2}=\sum\left(n_{i}-3\right)\left(z_{i}-\bar{z}\right)^{2}$

is approximately $\chi^{2}$ distributed with $k-1$ degrees of freedom. A similar test can be obtained for the partial correlation coefficients if the degrees of freedom are reduced by the number of variables kept fixed. In this study this number is one (the time).
The 100(1- $\alpha)$ per cent confidence interval for the theoretical correlation coefficient can be obtained if we use the method for transformed variables presented above. If the variable $z$ is assumed to be asymptotically normal, we obtain

$$
\begin{aligned}
& P\left(\varsigma_{L}<\varsigma<\varsigma_{U}\right)=1-\alpha \text { where } \varsigma_{L}=\bar{z}-\frac{u_{1 / 2 \alpha}}{\sqrt{n-3}} \text { and } \\
& \varsigma_{U}=\bar{z}+\frac{u_{1 / 2 \alpha}}{\sqrt{n-3}}
\end{aligned}
$$

where $\mathrm{u}_{1 / 22}$ is the $(1-1 / 2 \alpha)$ quantile of a standardized normal variable. Returning back to $\rho$ and $r$, we

$P\left(\rho_{L}<\rho<\rho_{U}\right) \approx 1-\alpha$, where $\rho_{L}=\frac{e^{2 \varsigma_{L}}-1}{e^{2 \varsigma_{L}}+1}$ and

$\rho_{U}=\frac{e^{2 \varsigma_{U}}-1}{e^{2 \varsigma_{U}}+1}$

The nonlinear transformation yields confidence intervals where the estimated correlation coefficient is not at the centre of the interval, but the confidence intervals are always included in the admissible interval $(-1,1)$.

In general, let the notations for the correlation coefficients between the $x, y$ and time $(t)$ be $r_{x, y}, r_{x, t}$ and $r_{y, t}$, respectively. The partial correlation coefficient between $x$ and $y$, when the time is kept fixed, is

$$
r_{x, y, t}=\frac{r_{x, y}-r_{x, t} r_{y, t}}{\sqrt{\left(1-r_{x, t}^{2}\right)\left(1-r_{y, t}^{2}\right)}}
$$

and an approximate formula of its standard error is

$$
\operatorname{SE}\left(r_{x, y ; t}\right)=\frac{1-\rho_{x, y ; t}^{2}}{\sqrt{n}}
$$

Partial correlation coefficients may differ markedly from the ordinary correlation coefficients and even have different signs. An alternative attempt to eliminate the effects of the time-dependent factors is to consider regional cross-sectional data.

The test of a correlation coefficient demands samples of rather large sizes and consequently, significant results are obtained only for long time series or for large numbers of observation pairs in cross-sectional studies. This property can by stressed in the following way. The significance test of correlation coefficients against the null hypothesis that the theoretical correlation coefficient is zero yields a critical region proportional to $\mathrm{n}^{-1 / 2}$. In our studies, the time series based on decade data consist of (at most) 21 sub-periods and the cross-sectional data consist of (at most) 25 counties. If we assume a $5 \%$ significance level and test against the null hypothesis $\rho=0$, we obtain for $n=20$ and for $n=25$ the critical values 0.44 and 0.39 , respectively. In addition, we have to note that for some counties the number of subperiods is markedly below 21 and for some periods the number of counties is less than 25 .

Correlations between the twinning rate and the rates of higher multiple maternities. For Sweden the correlation coefficients between $W$ and $R$ and the transformed triplet rate $\left(R_{H}\right)$ based on 10-year data $(n$ $=21$ ) are both 0.866 . These estimates are statistically 
significant. The temporal variation in $W$ and $R_{H}$ are similar, showing strong decreasing trends (Figure 1). If we eliminate the time effect and consider the partial correlation coefficients between $W$ and $R$ and between $W$ and the $R_{H}$, we obtain the reduced values 0.620 and 0.622 , respectively (Table 1 ). The correlation coefficients are reduced, but they are still statistically significant. The correlation coefficients between $W$ and $Q$ and the transformed quadruplet rate $\left(Q_{H}\right)$ based on the 10 -year data $(n=21)$ are 0.700 and 0.648 , respectively. These correlations are statistically significant. After elimination of the time effect, we obtain the partial correlation coefficients 0.217 and 0.147 , respectively. Neither of these is statistically significant. These results support the hypothesis that the ordinary correlation coefficients obtained are at least partly influenced by some external time-dependent factors.
In Table 1 we present the ordinary correlations between time, the $W, R$ and $R_{H}$, based on time-series data for the 25 counties in Sweden for 1751-1960. Sweden as a whole is included for comparison. Marked discrepancies from the expected positive correlation between $W$ and $R$ (and $R_{H}$ ) can be seen. For the counties of Älvsborg, Kronoberg, Jönköping, Västerbotten and Jämtland we obtain negative correlation coefficients. Strong positive correlation coefficients (more than 0.4) are obtained for the counties of Örebro, Kalmar, Östergötland, Värmland, Kopparberg and Skaraborg. The lack of a systematic pattern in the correlations between time and $W, R$ and $R_{H}$ supports the hypothesis that the rates are influenced by some common external factors and that these have different effects in different regions. Therefore, we try to eliminate the effect of these factors and calculate the partial correlations between $W$ and $R$ and $R_{H}$ when time is

Table 1

Correlations for the Period 1751-1960 Between Time $(t)$, the Triplet Rate $(R)$, the Transformed Triplet Rate $\left(R_{H}\right)$ and the Twinning Rates $(W)$ in the Counties of Sweden.

Sweden as a whole is included as a comparison. The $\chi^{2}$ values (Hald, 1967) measure the regional heterogeneity in the correlation coefficients.

\begin{tabular}{|c|c|c|c|c|c|c|c|c|}
\hline & & \multicolumn{7}{|c|}{ Correlation coefficients } \\
\hline & & \multicolumn{5}{|c|}{ Ordinary } & \multicolumn{2}{|c|}{ Partial } \\
\hline & & $t$ vs. $R$ & $t$ vs. $R_{H}$ & $t$ vs. $W$ & $R$ vs. $W$ & $R_{H}$ vs. $W$ & $R$ vs. $W$ & $R_{H}$ vs. $W$ \\
\hline SWEDEN & & -0.896 & -0.903 & -0.770 & 0.866 & 0.866 & 0.620 & 0.622 \\
\hline Counties & Codes & & & & & & & \\
\hline Stockholm city & A & -0.624 & -0.651 & -0.894 & 0.589 & 0.607 & 0.087 & 0.073 \\
\hline Stockholm county & B & -0.610 & -0.634 & -0.915 & 0.659 & 0.682 & 0.315 & 0.326 \\
\hline Uppsala & C & -0.743 & -0.757 & -0.821 & 0.663 & 0.681 & 0.141 & 0.161 \\
\hline Södermanland & $\mathrm{D}$ & -0.613 & -0.658 & -0.892 & 0.679 & 0.690 & 0.369 & 0.304 \\
\hline Östergötland & $E$ & -0.580 & -0.350 & -0.589 & 0.680 & 0.656 & 0.513 & 0.594 \\
\hline Jönköping & $\mathrm{F}$ & 0.135 & 0.146 & 0.048 & -0.265 & -0.272 & -0.274 & -0.282 \\
\hline Kronoberg & G & -0.502 & -0.543 & 0.284 & -0.373 & -0.189 & -0.278 & -0.044 \\
\hline Kalmar & $\mathrm{H}$ & -0.418 & -0.445 & -0.293 & 0.576 & 0.496 & 0.522 & 0.554 \\
\hline Gotland & I & -0.550 & -0.470 & -0.887 & 0.648 & 0.504 & 0.416 & 0.213 \\
\hline Blekinge & $\mathrm{K}$ & -0.723 & -0.723 & -0.081 & 0.246 & 0.261 & 0.271 & 0.293 \\
\hline Kristianstad & $\mathrm{L}$ & -0.469 & -0.495 & 0.182 & 0.359 & 0.386 & 0.511 & 0.558 \\
\hline Malmöhus & $\mathrm{M}$ & -0.528 & -0.514 & -0.192 & 0.362 & 0.366 & 0.312 & 0.317 \\
\hline Halland & $\mathrm{N}$ & -0.545 & -0.353 & -0.367 & 0.208 & 0.102 & 0.011 & -0.032 \\
\hline Gothenburg\&Bohus & 0 & -0.304 & -0.314 & -0.620 & 0.417 & 0.408 & 0.306 & 0.285 \\
\hline Älvsborg & $\mathrm{P}$ & -0.676 & -0.690 & 0.039 & -0.240 & -0.263 & -0.290 & -0.326 \\
\hline Skaraborg & $\mathrm{R}$ & -0.748 & -0.761 & -0.267 & 0.496 & 0.473 & 0.463 & 0.431 \\
\hline Värmland & S & -0.627 & -0.647 & -0.596 & 0.478 & 0.492 & 0.168 & 0.174 \\
\hline Örebro & $\mathrm{T}$ & -0.209 & -0.294 & -0.761 & 0.724 & 0.782 & 0.891 & 0.901 \\
\hline Västmanland & $U$ & -0.627 & -0.616 & -0.935 & 0.643 & 0.639 & 0.203 & 0.224 \\
\hline Kopparberg & W & -0.279 & -0.290 & -0.637 & 0.553 & 0.606 & 0.507 & 0.571 \\
\hline Gävleborg & $x$ & -0.685 & -0.698 & -0.946 & 0.687 & 0.729 & 0.164 & 0.295 \\
\hline Västernorrland & $Y$ & -0.338 & -0.302 & -0.557 & 0.316 & 0.300 & 0.164 & 0.166 \\
\hline Jämtland & Z & -0.252 & 0.024 & -0.705 & 0.132 & -0.053 & -0.067 & -0.051 \\
\hline Västerbotten & $A B$ & -0.645 & -0.550 & 0.139 & -0.146 & -0.150 & -0.074 & -0.088 \\
\hline Norrbotten & $C D$ & -0.264 & -0.129 & 0.047 & 0.119 & 0.126 & 0.137 & 0.133 \\
\hline $\begin{array}{l}\text { Test of regional } \\
\text { heterogeneity }\end{array}$ & $\chi_{24}^{2}$ & 141.1 & 137.2 & 290.9 & 112.9 & 110.7 & 52.49 & 55.53 \\
\hline
\end{tabular}




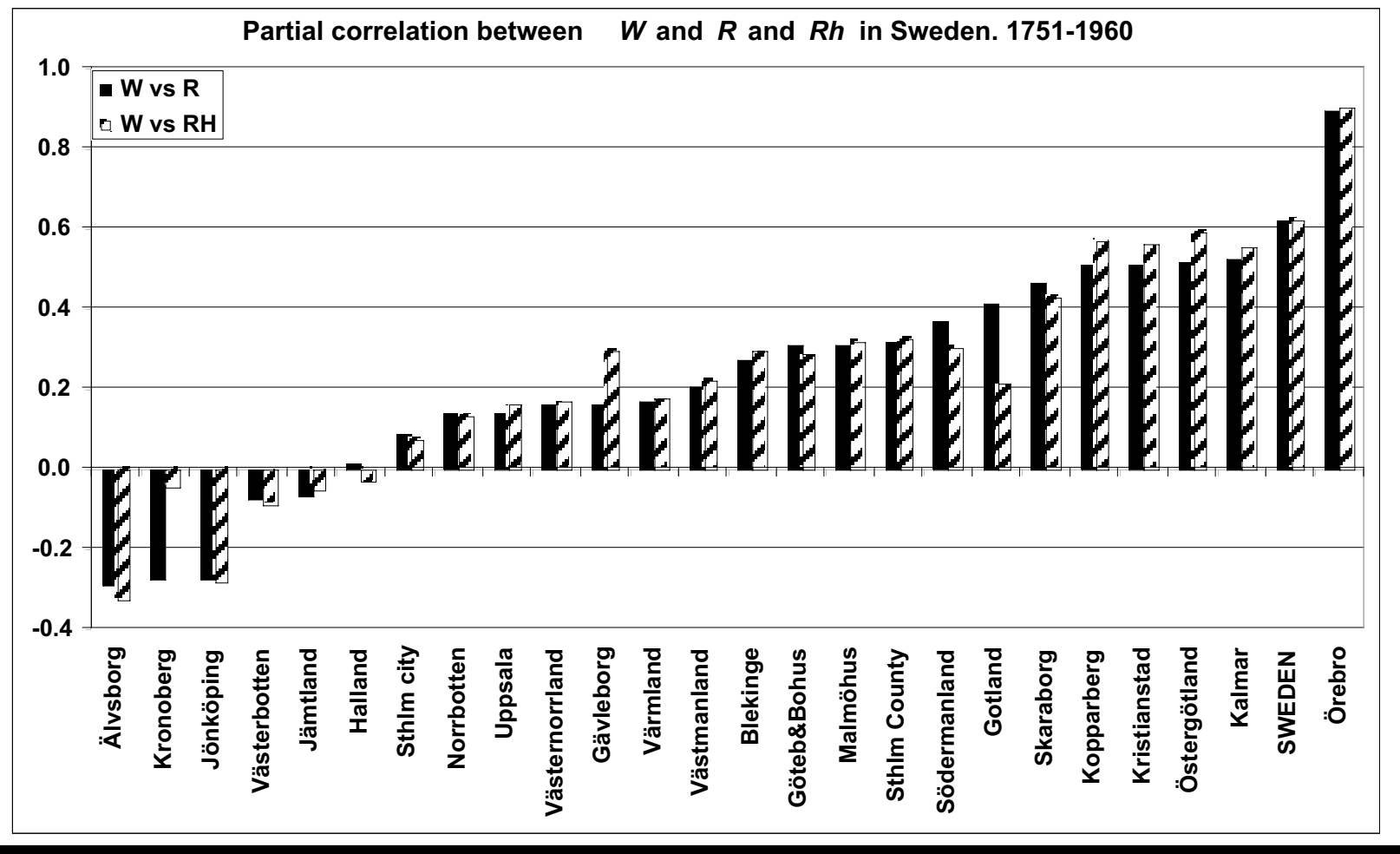

\section{Figure 3}

Partial correlation coefficients between the twinning rate $(\mathrm{W})$ and the triplet rate $(\mathrm{R})$ and the transformed triplet rate $(\mathrm{Rh})$, when time is kept fixed, for the different counties of Sweden.

The counties are arranged according to increasing levels of the correlation between $\mathrm{W}$ and $\mathrm{R}$. No systematic association between the $\mathrm{W}$ and the $\mathrm{R}$ can be identified. Marked discrepancies from the expected positive correlation between W and R (and Rh) can be seen. For comparison, the correlation coefficients for Sweden are included.

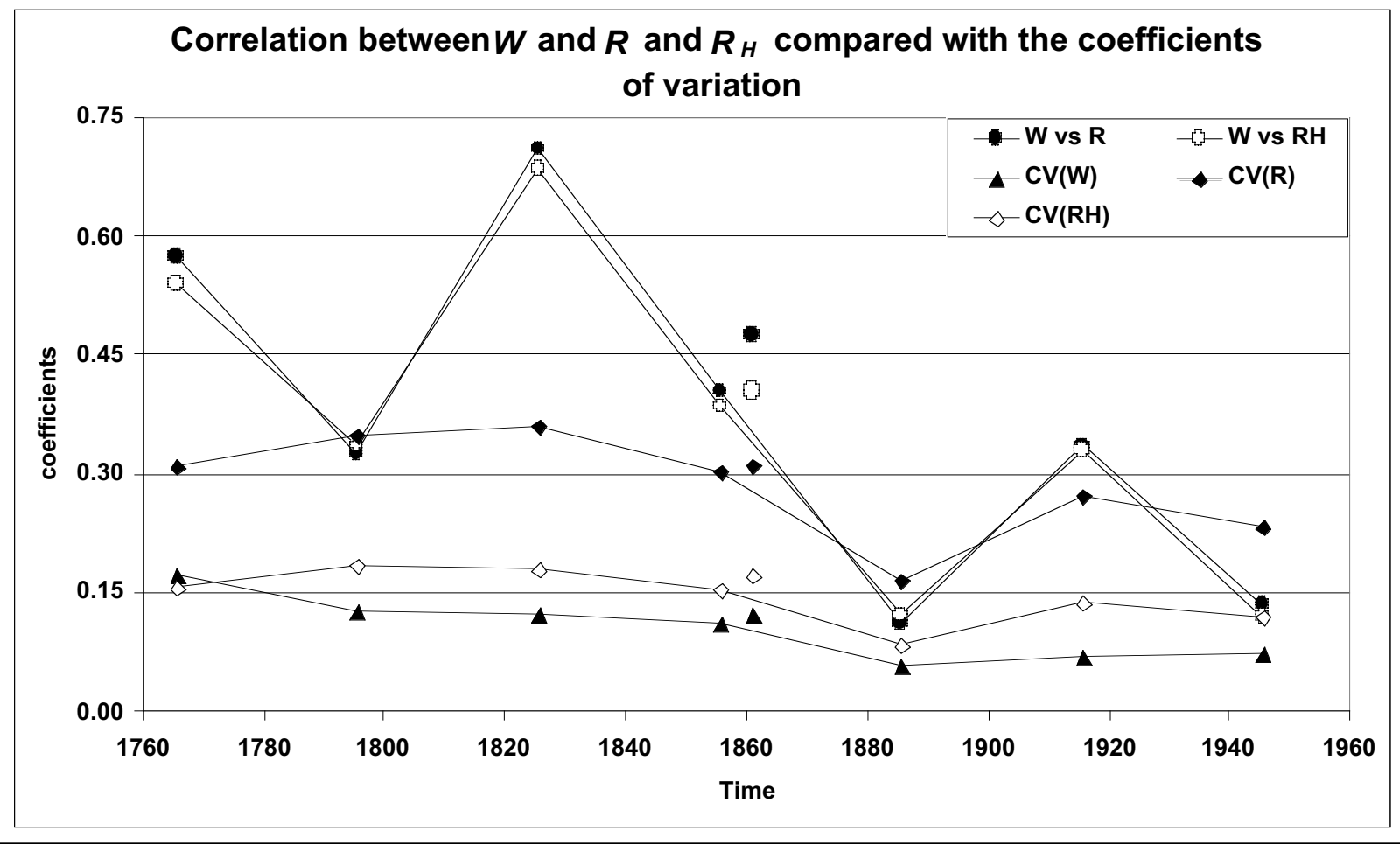

Figure 4

Correlation between the regional twinning rate ( $M$ and the regional observed and transformed triplet rates $\left(R\right.$ and $R_{4 \prime}$ respectively).

The numerical values are given in Table 2. The isolated points are based on the data presented by Berg (1880) for the period 1849-1873. Only the correlation coefficients for the periods 1751-1780, 1811-1840, 1841-1870 (for $R$ ) and 1849-1873 are statistically significant $(P<.05)$. The interregional variations in $W, R$ and $R_{H}$ are measured by the coefficients of variation (CV). 
Table 2

Correlation Between the Regional Twinning Rate $\left(W\right.$ and the Regional Observed and Transformed Triplet Rates ( $R$ and $R_{H^{\prime}}$ Respectively) for the Seven 30-Year Periods Between 1751 and 1960.

The interregional variations are measured by the coefficients of variation (CV). As a comparison, the correlation coefficients and CVs based on Berg's data (1880) for the period 1849-1873 are included. Only the correlation coefficients for the periods 1751-1780, 1811-1840, 1841-1870 (for $R$ ) and $1849-1873$ are statistically significant.

\begin{tabular}{lccccc}
\hline Period & \multicolumn{2}{c}{ Correlation coefficient } & \multicolumn{3}{c}{ Coefficient of variation } \\
\hline & $W$ vs $R$ & $W$ vs $R_{H}$ & $W$ & $R$ & $R_{H}$ \\
\hline $1751-1780$ & 0.574 & 0.539 & 0.172 & 0.309 & 0.157 \\
$1781-1810$ & 0.326 & 0.335 & 0.127 & 0.350 & 0.186 \\
$1811-1840$ & 0.710 & 0.685 & 0.123 & 0.360 & 0.180 \\
$1841-1870$ & 0.405 & 0.386 & 0.112 & 0.304 & 0.154 \\
$1849-1873$ & 0.475 & 0.405 & 0.123 & 0.311 & 0.172 \\
$1871-1900$ & 0.112 & 0.121 & 0.060 & 0.166 & 0.084 \\
$1901-1930$ & 0.336 & 0.330 & 0.071 & 0.274 & 0.138 \\
$1931-1960$ & 0.136 & 0.120 & 0.074 & 0.232 & 0.120 \\
\hline
\end{tabular}

kept fixed. The partial correlations obtained are also given in Table 1. We still observe no general pattern. For the counties of Jönköping, Kronoberg, Älvsborg, Jämtland and Västerbotten the partial correlation coefficients between $W$ and $R$ and $R_{H}$ are negative. In contrast, we observe very high positive correlation coefficients for the counties of Örebro, Östergötland, Kopparberg, Kristianstad and Kalmar. In general, $R$ and $R_{H}$ give very similar results and therefore no transformation is necessary. However, the transforma- tion is valuable for descriptive comparisons, because the $W$ and the $R_{H}$ are on the same scale.

Using the $\chi^{2}$ test described above we can test the regional heterogeneity between the correlation coefficients. We obtain statistically significant differences for all the correlation coefficients (see Table 1). The regional heterogeneity, according to the $\chi^{2}$ test described above, is more marked in $W$ than in $R$ or $R_{H}$. This regional heterogeneity is still observable when partial correlation coefficients are compared, but the heterogeneity is reduced. In Figure 3 we

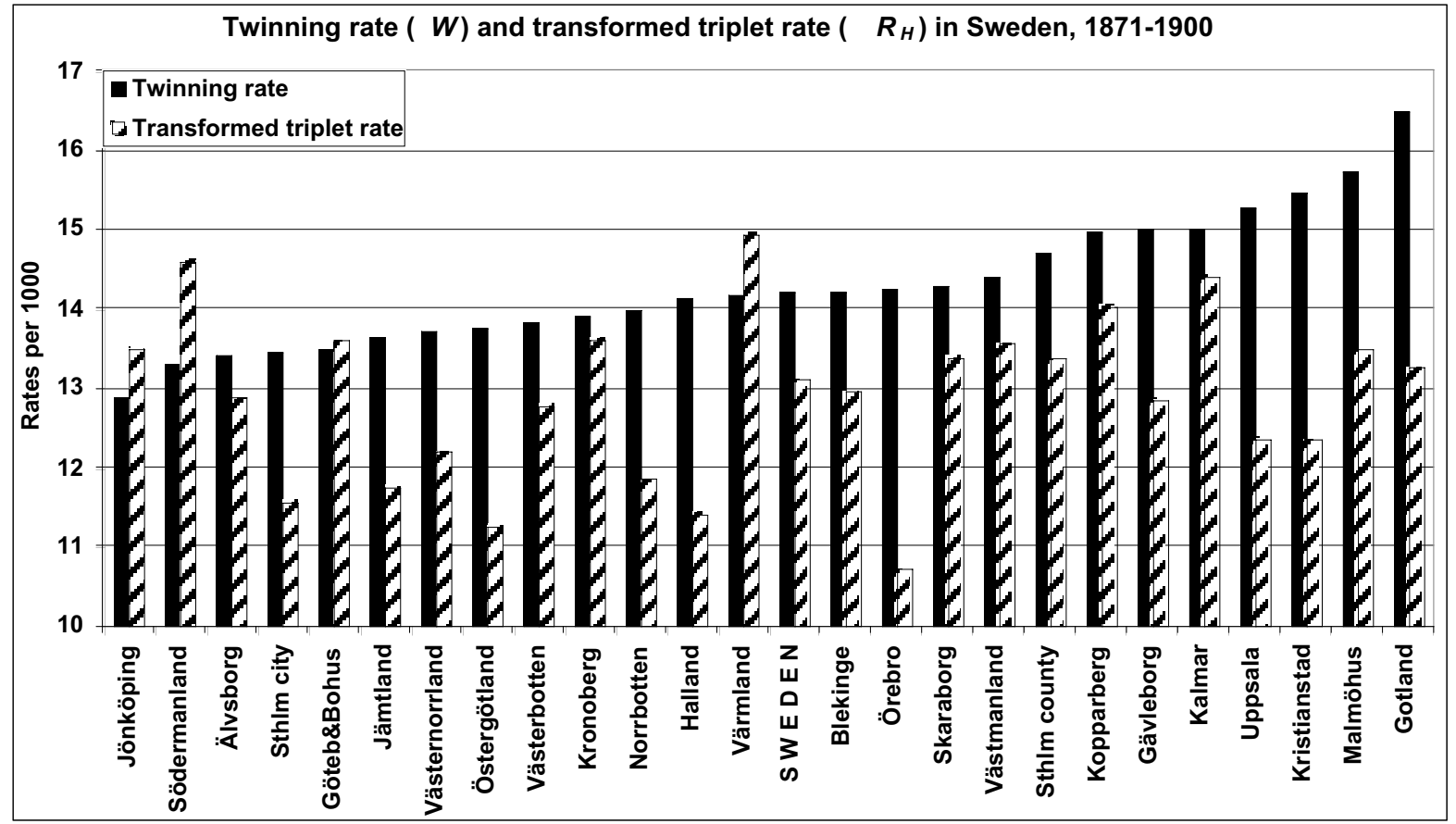

\section{Figure 5}

Comparison between the twinning rate $\left(W\right.$ and the transformed triplet rate $\left(R_{H}\right)$ for the 25 counties for the period 1871-1900.

The counties are arranged according to the increasing levels of the twinning rate. The correlation coefficient between $W$ and $R_{H}$ is only 0.121 , being insignificant. We observe obvious exceptions from the expected patterns that the $W$ should be slightly higher than the $R_{H}$ and that counties with high twinning rates have high triplet rates and vice versa. 
present the partial correlation coefficients in increasing order. The partial correlation coefficients vary between -0.290 for $R\left(-0.326\right.$ for $\left.R_{H}\right)$ and 0.891 (0.901) and no systematic association between the twinning and the triplet rates can be identified.

The temporal effects on the correlation between the multiple rates can also be eliminated if we consider the cross-sectional data distributed across the 25 counties in Sweden. We consider Berg's data for the period 1849-1873 and the official data for the seven 30 -year periods presented in Table 2. For these periods we calculate cross-sectional correlation coefficients. Only the correlation coefficients for the periods 1751-1780, 1811-1840, 1841-1870 (for $R_{H}$ ) and $1849-1873$ are statistically significant $(P<.05)$. After 1810, a decreasing tendency can be observed. The interregional variations in $W, R$ and $R_{H}$ are measured by the coefficients of variation $(\mathrm{CV})$ and these data are also presented in Table 2 and Figure 4. We observe a slight decrease in regional heterogeneity in the $W$. This finding is discussed in greater detail in Fellman and Eriksson (2004). Consequently, our opinion is that the use of regional correlation coefficients in cross-sectional data and partial correlation coefficients in the time series have at least partly eliminated the effects of the time-dependent factors.

In Figure 5 we compare $W$ and $R_{H}$ for 25 counties for the period 1871-1900; that is, a 30-year period with the lowest correlation coefficient. We observe obvious exceptions from the expected pattern that the W should be slightly higher than the $R_{H}$ and that counties with high twinning rates have high triplet rates and vice versa. For the counties of Örebro, Gotland, Kristianstad, Uppsala, Malmöhus, and Halland we observe high $W$ and low $R_{H}$ (a difference of more than 2.5 per mille units) and for the counties of Södermanland, Värmland, Jönköping and Gothenburg\&Bohus low $W$ and high $R_{H}$ (negative difference). In fact, for the period 1871-1900 the correlation coefficient between $W$ and $R_{H}$ is only 0.121 , being insignificant.

\section{Discussion}

Although Hellin's law is only a rule of thumb, we also considered, besides $R$, the transformed rate $R_{H}$. Comparing the associations between $W$ and $R$ and $R_{H}$, we observe no great discrepancies. Of the rates $\mathrm{R}$ and $R_{H}$, the latter has the advantage that it can be presented together with $W$ on the same scale. The rates $Q$ and $Q_{H}$ are only studied superficially, because quadruplet maternities are such rare events that more detailed analyses give uncertain results. The rates of quadruplet maternities in Sweden (1751-1850) were about 6 per million maternities, but after 1850 went down strongly to values around 2 during the next hundred years. After 1950 the rate is more than 7 per million, mainly due to the use of ovulation stimulants and other artificial reproduction methods, as discussed above. In fact, our whole series for Sweden contains only 112 quadru- plet sets. In combined data from Europe and the USA, Bulmer (1970) noted a frequency of 1.7 quadruplet sets per million maternities. Parisi and Caperna (1981) confirmed these values for Italy during the overall period 1868-1977, but peaks in excess of 2.6 quadruplet and quintuplet maternities were reached in the periods 1930-1944 and 1970-1977. In Sweden there has also been a steeper downward secular trend of triplet and quadruplet rates than of the twinning rate (cf. Figure 1 and see Eriksson, 1973).

The lack of a systematic association between the rates of multiple maternities indicates that the rates may be influenced by external, time-dependent, and still unknown factors. In order to remove the effect of these factors, one possibility was to consider time as a proxy variable for these and eliminate the effect of the unknown factors by studying partial correlation coefficients between $W$ and $R$ for the different counties when time is kept fixed. We considered only the period 1751-1960 because, during this period and particularly after the 1780s, with more efficient registration of stillborn multiples (Eriksson et al., 1988), a general decreasing tendency can be observed and the effect of external variables can be assumed to be monotonic. After the 1920s the $W$ went down strongly, reaching a minimum below 9 per 1000 in the 1970s.

Multiple ovulations and multiple conceptions seem to be much more common than multiple maternities, because intrauterine deaths occur extensively and particularly in pregnancies with multiple embryos or fetuses (Boklage, 1995; Landy \& Keith, 1998) in which the volume of circulating blood required is considerably higher than in singleton pregnancies (Kauppinen, 1967). The increased urbanization and modern life with more sedentary occupations among mothers has caused a deterioration of physical condition. This may have decreased the blood circulation and the risk that multiple gestations will end in singleton maternities or total abortion (Eriksson, 1964, 1973). This hypothesis is consistent with our findings that both the gross and the age-specific rates of multiple maternities - and particularly the triplet and quadruplet rates - have been higher in rural than in urban populations (Eriksson, 1964, 1973; Eriksson \& Fellman, 1973). In addition, there is evidence that women of mixed ancestry, due to intermarriage between populations with different dizygotic twinning rates, produce fewer twins than would be expected if gene action were additive (Bulmer, 1970; Eriksson, 1962, 1964, 1973; Eriksson \& Fellman, 2004; Morton et al., 1967).

The rates of multiple maternities tend to decrease with a higher degree of urbanization. The most probable reasons for the lower twinning rates in urban populations are the lower maternal age, the lower mean parity, the more frequent outmarriage, and the fact that, owing to more sedentary and monotonous work leading to poorer physical capacity, among other things, the mothers in urban areas are less well- 
fitted to carry through pregnancies with multiple embryos or fetuses. If the rate of spontaneous abortions in an early phase of gestation in triplet conceptions was higher than in twins, this could also lead to the observed frequency of triplet maternities being less than those expected from Hellin's formula.

In a summary of reports of triplet sets from different populations by Little and Thompson (1988), the only excesses of triplets in comparison with twins were found in Nigeria and in Papua. However, the series were very small. Among the eastern Swedes on the Åland Islands (1653-1949) the observed triplet rate $(37.5$ per 100,000$)$ was almost the same as the expected $(36.9$ per 100,000$)$. Both the twinning and the triplet rates showed strong secular declines (Eriksson, 1973). In this study an excess of observed over expected numbers of triplet maternities was found for the period 1871-1900 in four counties, Södermanland, Jönköping, Gothenburg\&Bohus and Värmland (Figure 5). In all the other counties and for Sweden as a whole there were fewer triplet maternities than expected. The correlation decreasing with time between $W$ and $R$ may be an indication of decreasing mean maternal age and parity. They both have a stronger effect on the triplet rate than on the twinning rate (Eriksson, 1990; Eriksson et al., 1995).

Our studies on the trends in twin and triplet maternities in Finland, comparing rural communities with towns between 1860 and 1959, also showed that during the period 1905-1955 the rates in rural communities clearly and steadily increased, while those in town populations, though at all times lower and more erratic, had a smaller increase (Eriksson \& Fellman, 1973). In the typically agrarian Finland, urbanization and industrialization began about one to two generations later than in Sweden (Eriksson \& Fellman, 1973) but was given a substantial boost after World War II in connection with Finnish compensations for war damage to the Soviet Union.

Our summarizing conclusion is that, after elimination of exogenous factors, the correlation between $W$ and $R$ is moderate.

\section{Acknowledgment}

This work was supported by grants from the 'Liv och Hälsa' Foundation and from the Finnish Society of Sciences and Letters. We are very grateful to Dr Åke Nilsson and his co-workers at Statistics Sweden for cooperation in sending us information on details of twin maternities.

\section{References}

Allen, G. (1960). A differential method for estimation of type frequencies in triplets and quadruplets. American Journal of Human Genetics, 12, 210-224.

Berg, F. T. (1880). Om flerfostriga barnsbörder. (On multiple maternities, in Swedish.) Hygiea (Stockholm), $42,331-342$.
Boklage, C. E. (1995). The frequency of natural twin conceptions. In L. G. Keith, E. Papiernik, D. M. Keith \& B. Luke (Eds.), Multiple Pregnancy: Epidemiology, Gestation and Perinatal Outcome (pp. 41-50). New York: Parthenon.

Bulmer, M. G. (1970). The Biology of the Twinning in Man, Chapters 5-6 (pp. 205). Oxford - London: Oxford University Press.

Eriksson, A. W. (1962). Variations in the human twinning rate. Acta Genetica (Basel), 12, 242-250.

Eriksson, A. W. (1964). Pituitary gonadotrophin and dizygotic twinning. Lancet, 2, 1298-1299.

Eriksson, A. W. (1973). Human twinning in and around the Åland Islands. Commentationes Biologicae, 64, $1-154$.

Eriksson, A. W. (1990). Twinning in families of triplets. Acta Genetica Medica Gemellologica, 39, 279-293.

Eriksson, A. W., Abbott, C., Kostense, P. J., \& Fellman, J. O. (1995). Secular changes of twinning rates in Nordic populations. Acta Genetica Medica Gemellologica, 44, 141-161.

Eriksson, A. W., \& Fellman. J. (1973). Differences in the twinning trends between Finns and Swedes. American Journal of Human Genetics, 25, 141-151.

Eriksson, A. W., \& Fellman, J. (2004). Demographic analysis of the temporal and regional variation in the rates of multiple maternities in Sweden since 1749. Human Biology, (in press).

Eriksson, A.W., Bressers, W. M. A., Kostense P. J., Pitkänen, K. J., Mielke, J. H., Jorde, L. B., Tas, R. F. J., \& Fellman, J. O. (1988). Twinning rate in Scandinavia, Germany and the Netherlands during years of privation. Acta Geneticae Medicae et Gemellologiae, 37, 277-297.

Fellman, J. O., \& Eriksson, A. W. (1993). Biometric analysis of multiple maternities in Finland, 1881-1990, and Sweden since 1750. Human Biology, $65,463-479$.

Fellman, J., \& Eriksson, A. W. (2003). Temporal differences in the regional twinning rates in Sweden after 1750. Twin Research, 6, 183-191.

Fellman, J., \& Eriksson, A. W. (2004). The convergence of the regional twinning rates in Sweden, 1751-1960. (In manus).

Hald, A. (1967). Statistical Theory with Engineering Applications, Chapters 19-20 (pp. 783), (1952: 7th printing 1967). New York: John Wiley.

Hellin, D. (1895). Die Ursache der Multiparität der uniparen Tiere überhaupt und der Zwillingsschwangerschaft beim Menschen insbesondere (pp. 70). München: Seitz \& Schauer.

Kauppinen, M. A. (1967). The correlation of maternal heart volume with birth weight of the infant and prematurity. Acta Obstetricia et Gynecologica Scandinavica, 46 (Suppl 6), 1-128. 
Kendall, M. G., \& Stuart, A. (1967). The Advanced Theory of Statistics: Vol. 2. Chapter 26. (2nd ed., pp. 690). London: Charles Griffin.

Landy, H. J., \& Keith, L. G. (1998). The vanishing twin: a review. Human Reproduction Update, 4, 177-183.

Little, J., \& Thompson, B. (1988). Descriptive epidemiology. In I. MacGillivray, D. M. Campbell \& B. Thompson (Eds.), Twinning and Twins (pp. 34-92). Chichester: John Wiley.
Morton, N. E., Chung, C. S., \& Mi, M. P. (1967). Genetics of interracial crosses in Hawaii. Monographs in Human Genetics: 3. Basel, Switzerland: Karger.

Parisi, P., \& Caperna, G. (1981). The changing incidence of twinning: one century of Italian statistics. In L. Gedda, P. Parisi, \& W. E. Nance (Eds.), Twin Research 3, Part A, Twin Biology and Multiple Pregnancy (pp. 35-48). New York: Alan R. Liss. 